\title{
Mitotic figure counts are significantly overestimated in resection specimens of invasive breast carcinomas
}

Hans-Anton Lehr ${ }^{1}$, Candice Rochat ${ }^{1}$, Cornelia Schaper ${ }^{2}$, Antoine Nobile $^{1}$, Sherien Shanouda ${ }^{1}$, Sandrine Vijgen ${ }^{1}$, Arnaud Gauthier ${ }^{1}$, Ellen Obermann ${ }^{3}$, Susana Leuba ${ }^{1}$, Marcus Schmidt ${ }^{4}$, Curzio Ruegg $C^{5}$, Jean-Francois Delaloye ${ }^{6}$, Nectaria Simiantonaki ${ }^{7}$ and Stephan C Schaefer 8

${ }^{1}$ Institute of Pathology, Centre Hospitalier Universitaire Vaudois, Lausanne, Switzerland;

${ }^{2}$ Institute of Pathology, Johannes Gutenberg University, Mainz, Germany; ${ }^{3}$ Institute of Pathology, University of Basel, Basel, Switzerland; ${ }^{4}$ Department of Gynecology, Johannes Gutenberg University, Mainz, Germany; ${ }^{5}$ Department of Medicine, Faculty of Sciences, University of Fribourg, Fribourg, Switzerland; ${ }^{6}$ Department of Gynecology and Obstetrics, Centre Hospitalier Universitaire Vaudois, Lausanne, Switzerland; ${ }^{7}$ Institute of Pathology, Klinikum Gummersbach, Gummersbach, Germany and ${ }^{8}$ Institute of Pathology, Inselspital, Bern, Switzerland

\begin{abstract}
Several authors have demonstrated an increased number of mitotic figures in breast cancer resection specimen when compared with biopsy material. This has been ascribed to a sampling artifact where biopsies are (i) either too small to allow formal mitotic figure counting or (ii) not necessarily taken form the proliferating tumor periphery. Herein, we propose a different explanation for this phenomenon. Biopsy and resection material of 52 invasive ductal carcinomas was studied. We counted mitotic figures in 10 representative high power fields and quantified MIB-1 immunohistochemistry by visual estimation, counting and image analysis. We found that mitotic figures were elevated by more than three-fold on average in resection specimen over biopsy material from the same tumors ( $20 \pm 6$ vs $6 \pm 2$ mitoses per 10 high power fields, $P=0.008)$, and that this resulted in a relative diminution of post-metaphase figures (anaphase/telophase), which made up $7 \%$ of all mitotic figures in biopsies but only $3 \%$ in resection specimen $(P<0.005)$. At the same time, the percentages of MIB-1 immunostained tumor cells among total tumor cells were comparable in biopsy and resection material, irrespective of the mode of MIB-1 quantification. Finally, we found no association between the size of the biopsy material and the relative increase of mitotic figures in resection specimen. We propose that the increase in mitotic figures in resection specimen and the significant shift towards metaphase figures is not due to a sampling artifact, but reflects ongoing cell cycle activity in the resected tumor tissue due to fixation delay. The dwindling energy supply will eventually arrest tumor cells in metaphase, where they are readily identified by the diagnostic pathologist. Taken together, we suggest that the rapidly fixed biopsy material better represents true tumor biology and should be privileged as predictive marker of putative response to cytotoxic chemotherapy. Modern Pathology (2013) 26, 336-342; doi:10.1038/modpathol.2012.140; published online 5 October 2012
\end{abstract}

Keywords: biopsy; breast cancer; cell proliferation; metaphase; mitotic figure count; proliferative activity; resection specimen

For some patients to benefit from aggressive chemotherapy for invasive breast carcinoma, many patients are currently being treated with little or

Correspondence: Professor Hans-Anton Lehr, Institute of Pathology, Centre Hospitalier Universitaire Vaudois, Rue du Bugnon 25, Lausanne CH-1011, Switzerland.

E-mail: hansanton.lehr@yahoo.de

Received 30 May 2012; revised 2 July 2012; accepted 3 July 2012; published online 5 October 2012 no benefit. Enormous effort is hence being directed towards the identification of those patients who will benefit from chemotherapy and those who will not. As cytotoxic chemotherapy targets proliferating cells, ${ }^{1}$ pathologists focus on the proliferative activity of tumors, as assessed by mitotic figure counts or by the immunohistochemical detection of the cell-cycle-specific antigen Ki-67. ${ }^{2}$ In fact, the mitotic figure count is a key element of the timehonored grading of invasive breast carcinomas and 
has been routinely assessed in breast carcinomas for more than five decades. ${ }^{3,4}$ Since the advent of coreneedle biopsies to diagnose breast tumors, many authors have compared the histological findings obtained in biopsy material with the findings obtained in resection specimen. As far as the tumor grade is concerned, these studies have reported a tendency to upgrade carcinomas in resection specimen when compared with the initial diagnosis made on the biopsy material, and several authors have noted that the upgrade in resection specimen is due solely or to a large extent to an increase in mitotic figure counts. ${ }^{5-11}$ Explanations for this phenomenon have been sought in intratumoral heterogeneity and in sampling artifacts, suggesting that the biopsy material is either (i) too small for proper mitotic figure counting or (ii) taken at random from anywhere within the tumor and not necessarily from the more proliferative tumor periphery. ${ }^{7-11}$ It has consequently been suggested that discrepancies in tumor grades could be reduced by increasing the number and the size of the biopsy cores, 9,10 but no data have ever been published in support of that idea. As a consequence of this concept, several authors have suggested that in case of grade discrepancies between biopsy and resection specimen, therapeutic decisions should be based on the grade obtained in the resection specimen. ${ }^{7,12}$

Herein, we propose a different explanation for the discrepancy in mitotic figure counts between biopsy and resection material. We assessed the proliferative activity of 52 invasive ductal carcinomas and confirm that the number of mitotic figures significantly increased in resection specimen by a factor of more than 3 over the counts in biopsy material, whereas at the same time, the pan-cell-cycle-specific marker MIB-1 (targeting Ki-67) yielded comparable results for biopsies and resection specimen. We also found that the proportion of post-metaphase figures (anaphase and telophase) among the counted mitotic figures in resection specimen is less than half of those found in biopsies. On the basis of these observations, we propose here that the delayed formalin fixation of resection specimen allows cell cycle activities to continue in the resected tissue for up to many hours, resulting in a preferential arrest of mitoses in metaphase, where they are readily identified by the pathologist. As a consequence of this reasoning, we propose that the mitotic figure count in the rapidly fixed biopsy cores better represents the tumor biology and should be used as a basis for therapeutic decisions.

\section{Materials and methods}

\section{Patients}

We selected 52 consecutive cases of invasive ductal carcinomas from the archives of the University Institute of Pathology at the Centre Hospitalier Universitaire Vaudois (CHUV, 3/2008-4/2009). Only those cases were chosen for which we disposed of paraffin blocks of true-cut biopsy material as well as of resection specimen (tumorectomies or mastectomies). The slides were assigned random codes from 1 to 104, which were used for the entire series of experiments. No patient identifiers were used for any of the different part of the study. The study protocol was approved by the local ethical committee (CEV-VD BB09-15).

\section{Mitotic Figure Counts}

Hematoxylin and eosin $(\mathrm{H} \& \mathrm{E})$ stainings were performed according to standard protocols on $4-\mu \mathrm{m}$ sections and used for mitotic figure counts. Mitotic figures were counted in 10 high power fields (Olympus BX-45 diagnostic microscope, $0.52 \mathrm{~mm}$ diameter of the high power field), corresponding to grade cut-offs for mitotic figure counts of 0-6 (grade 1), 7-14 (grade 2) and $\geqslant 15$ (grade 3). ${ }^{13}$ As in our daily diagnostic routine, the guidelines of van Diest et $a l^{14}$ were applied for the correct identification of mitotic figures. In biopsy material, areas were selected at random for mitotic figure counting where the high power field was filled with invasive carcinoma as much as possible. Ten complete high power fields could be counted in 47 of the 52 carcinomas, and in the remaining 5 cases, between 5 and 9 high power fields could be quantified. In resection specimen, we selected the proliferatively most active tumor periphery, but avoided hotspots. Mitotic figures were counted jointly by two observers under a double-headed diagnostic microscope (Olympus BX-45). Each mitotic figure was pointed out with an arrow in the microscope tube and only those mitotic figures were counted for which consensus was obtained by both the observers. We not only counted the total number of mitotic figures, but noted their phase of the cell cycle (prophase, prometaphase, metaphase, anaphase and telophase). In addition, we estimated approximately by visual estimation for each high power field the percentage of the field covered by tumor cells vs stroma and non-neoplastic ductules/ lobules.

\section{Immunohistochemistry}

Immunohistochemical assays were performed using an automated system from Ventana (Benchmal XL; Ventana, Tucson, AZ) on $4-\mu \mathrm{m}$ sections on superfrost slides after heat-induced epitope retrieval (30 min, EDTA buffer, proprietary information retained by Ventana). We used antibodies to Ki-67 (MIB-1, clone M7240; DAKO, Glostrup, Denmark; dilution 1:40). Quantification of MIB-1-immunostained slides was performed in three different ways. For the first one, we performed a visual estimation of the percentage of MIB-1-immunostained tumor cells, usually done at $\times 20$ magnification 
and assessing about 3-5 fields in the biopsy at random or in the proliferatively active tumor periphery in resection specimen, avoiding hotspots. In analogy to mitotic figure counting, MIB-1 estimation was performed by two observers (HAL and CR) using a discussion microscope, and consensus was obtained for all cases. In practice, both observers jointly scanned about five to ten $\times 20$ fields for about $20 \mathrm{~s}$, each, made mental notes of our MIB-1based proliferative index and then compared the results. In case of discrepancy, the case was discussed and a consensus index could be obtained in every case. In a second way, the percentage of MIB-1-positive tumor cells was counted by hand on high-quality color print-outs of five circular high power fields that were cropped from screen shots of virtual microscope slides (Hamamatsu NanoZoomer, Hamamatsu City, Japan). The high power fields had a diameter of $0.72 \mathrm{~mm}$, so that the 5 virtual high power fields compared with the surface of 10 microscopic high power fields. A minimum of 2000 tumor cells were counted for each case. Finally, we used the publicly available internet-based analysis software IMMUNORATIO to quantify the percentage of MIB-1-immunostained tumor nuclei (http://imtmicroscope.uta.fi/immunoratio/), uploading jpg files of the circular high power fields cropped from the virtual slides. Using inbuild map commands, this software identifies the surface covered by hematoxylin-counterstained (blue) nuclei and the surface covered by immunoreactive (brown) nuclei, and calculates an index of immunostained cells over the total cell number. ${ }^{15}$

\section{Photoshop-Based Image Analysis}

To investigate whether the discrepancy in mitotic figure counts between biopsy and resection material was less pronounced in larger biopsies, as proposed by some authors, ${ }^{9,10}$ we used Photoshop-based image analysis to quantify the size of the biopsy cylinders. For that purpose, the 52 biopsy slides were scanned using a commercially available flatbed scanner (EPSON perfection photo 3200) and the images opened in Photoshop (version CS3; Adobe Systems, San José, CA, USA) on a MacPro (Apple Computers, Cupertino, CA, USA). In analogy to previously published procedures, ${ }^{16}$ the biopsy cores were individually selected using the magic wand tool, and the number of pixels (indicated in the histogram window) was then used to calculate the surface of the cores (in $\mathrm{mm}^{2}$ ).

\section{Statistical Analysis}

The data obtained in the various analyses were manually entered in an excel file, and statistical analyses were calculated using a publicly available internet-based program (http://www.wessa.net/stat. wasp). We performed simple regression and Spear- man's analyses for the correlations between mitotic figure counts and immunohistochemical read-outs, and also performed bivariate paired $t$-tests to compare the results obtained in biopsies with those obtained in resection specimen. $P$-values of $<0.01$ were considered statistically significant.

\section{Results}

The number of mitotic figures per 10 high power fields ranged from 0 to 64 in biopsies and from 0 to 250 in resection specimen. Mean values \pm s.e.m. were $6 \pm 2$ in biopsies and $20 \pm 6$ in resection specimen $(P=0.00800)$. The increase in the number of mitotic figures from the biopsy to the resection specimen was 3.4-fold (s.e.m. 0.53), with a range of less than 1 to 12. The results are shown in graphic form in Figure 1 (left panel). When the numbers of mitotic figures were normalized to the surface of the high power fields taken up by tumor cells (vs stroma and non-neoplastic ductules/lobules), the numbers ranged from 0 to 80 for biopsies and 0 to 263 for resection specimen. Mean values \pm s.e.m. were $10 \pm 2$ in biopsies and $21 \pm 8$ in resection specimen $(P=0.00737)$. The increase in the number of mitotic figures from the biopsy to the resection specimen was 3 -fold (s.e.m. 0.4), with a range of less than 1 to 19 . The results are shown in graphic form in Figure 1 (right panel).

Motivated by the proposal by Harris et $a l^{9}$ that the discrepancy of mitotic figure counts between biopsies and resection specimen should be reduced in larger-sized biopsies, we correlated the relative increase of mitotic figure counts for each case pair with the size of the biopsy fragments, as assessed by Photoshop-based image analysis, which ranged from 2.5 to $104 \mathrm{~mm}^{2}$ (mean $9.6 \pm 2.0 \mathrm{~mm}^{2}$ s.e.m.), but could not identify such a correlation $(\rho=0.01859, P=0.8952)$.

We next analyzed whether the described increase in mitotic figures affects the overall SBR grades of the respective carcinomas. We found that based solely on the mitotic figure counts assessed in this systematic case review, 14 of the 52 carcinomas would have been upgraded in the resection specimen $(27 \% ; 6$ from grade 1 to grade 2 , and 8 from grade 2 to grade 3 ) and 5 were downgraded $(10 \% ; 4$ from grade 2 to grade 1 , and 1 from grade 3 to grade 2). The concordance rate between grades in biopsies and resection specimen was $63 \%$. Similar numbers were obtained when the mitotic figures are corrected for the tumor/stroma ratio: 15 carcinomas were upgraded (29\%; 6 from grade 1 to grade 2, and 9 from grade 2 to grade 3$)$ and 6 were downgraded $(11 \% ; 4$ from grade 2 to grade 1 ). Concordance rate: $60 \%$. If we compare this to the grades that were recorded in the original pathology reports concerning the respective cases, the observations were virtually identical: 15 cases experienced an upgrade $(29 \%$; 5 from grade 1 to grade 2 , and 10 from grade 2 to grade 

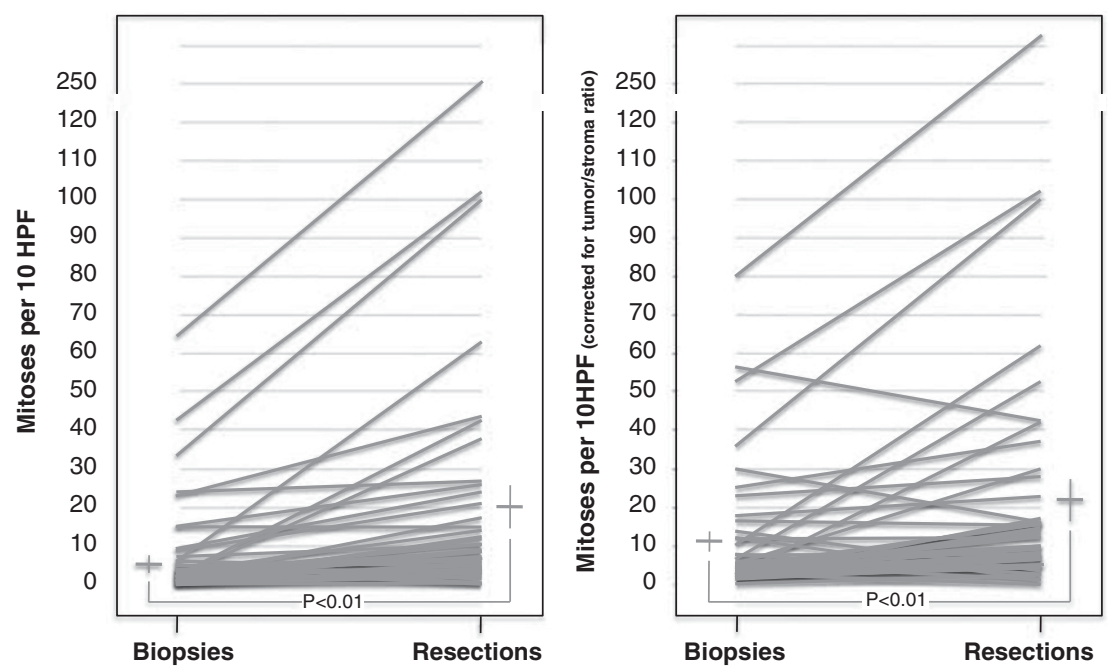

Figure 1 Mitotic figure counts in breast cancer biopsy material and resection specimen. Each line represents one of 52 invasive ductal carcinomas. Shown are mean values \pm s.e.m. Data are shown without (left panels) and with correction for the tumor/stroma ratio (right panel). Note that with only a few exceptions, the numbers of mitotic figures were significantly higher in resection specimen when compared with the respective biopsy material. $P<0.01$ (paired $t$-test).

$3)$ and 5 cases experienced a downgrade $(10 \% ; 3$ from grade 2 to grade 1 , and 2 from grade 3 to grade 2 ). Of the 15 cases that experienced an upgrade in the resection specimen, 14 were due to increased mitotic figure counts (and 1 was due to an overall reduced tubular architecture in the resection specimen), and of the 5 cases that experienced a downgrade, 3 were due to mitotic figure counts (and 2 due to increased overall tubular architecture in the resection specimen). The concordance rate was $62 \%$. These concordance rates are at the lower end of the spectrum (range 62 and $81 \%$ ) that has been reported by others. ${ }^{5-11}$

Next, we sub-classified the phases of the cell cycle of all the mitotic figures that we counted and found that there was a significant reduction in the percentage of post-metaphase figures (anaphase and telophase) from $7 \%$ of all mitotic figures in biopsies to $3 \%$ of all mitotic figures in resection specimen $(P<0.005$ paired Wilcoxon rank sum test with continuity correction).

We obtained MIB-1 immunohistochemical stains of all biopsies and resection specimen, and analyzed the percentage of MIB-1-stained tumor cells among all tumor cells by three different techniques: (i) visual estimation by two observers, (ii) counting on high-quality color print-outs of circular areas, $0.72 \mathrm{~mm}$ in diameter, cropped from digitized slides, and (iii) image analysis using IMMUNORATIO. ${ }^{15}$ We found that for all three techniques of MIB-1 quantification, there was no statistically significant difference in MIB-1 indices between biopsies and resection specimen: $20 \%$ vs $22 \%$ for visually estimated data (Figure 2, left panels), 22\% vs 22\% for counted data, and $14 \%$ vs $14 \%$ for image analysis (IMMUNORATIO), respectively. At the same time, the ratio of MIB-1 over mitotic figures was signifi- cantly $(P<0.02)$ reduced in resection specimen (Figure 2, right panel).

We next calculated simple linear regressions between mitotic figure counts (with and without correction for tumor/stroma ratio) and the percentage of MIB-1-positive nuclei (using the three distinct quantification techniques). The results for regression slopes (x), as well as $\rho$ - and $P$-values are shown in Table 1. As expected from the above findings, we noted a strong, highly significant correlation between the number of mitotic figures per 10 high power fields and the percentage of MIB1-positive tumor cells. The correlation was not improved by correcting mitotic figure counts for tumor/stroma ratios in the high power fields (Table 1). The $\rho$-values for the correlation between MIB-1 and mitotic figure counts correspond well to data published previously by us ${ }^{16}$ and by others. ${ }^{17}$ It also became apparent that as a consequence of the significantly higher mitotic figure counts in resection specimen, the slopes of the regression curves differed markedly between biopsies and resection specimen (Table 1), so that for a comparable MIB-1 index, the number of mitotic figures was significantly higher in the resection specimen than in the biopsies. These data are shown in graphic form in Figure 3.

Using paired $t$-tests, we found highly significant differences concerning the ratio of MIB-1 values over mitotic figure counts between biopsies and their corresponding resection specimen $(P$-values were $0.00169,0.000184$ and 0.0000951 for visually estimated, counted and computer-based MIB values over mitotic figures/10 high power fields, and 0.0196, 0.00995 and 0.00517 over mitotic counts normalized to the tumor/stroma ratio, respectively). 

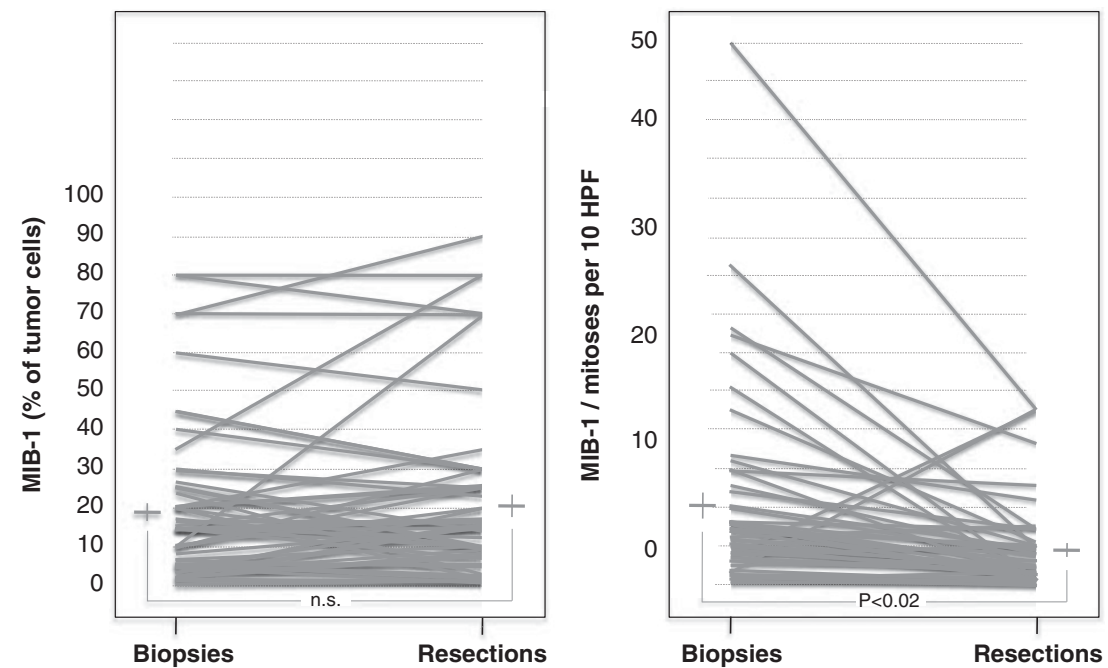

Figure 2 Proliferative index of breast carcinomas as assessed using MIB-1 immunohistochemistry in breast cancer biopsy material and resection specimen. Each line represents one of 52 invasive ductal carcinomas. MIB-1 immunohistochemistry was assessed by joint visual estimation by two observers. The left panel depicts the percentage of tumor cells labeled with MIB-1. The right panel depicts the ratio for each carcinoma of MIB-1 over the mitotic figure counts in 10 high power fields. Note that with the exception of a few cases, MIB-1 appears to be comparable in biopsy material and in resection specimen. However, the ratio of MIB-1 over mitotic figure counts is significantly reduced in resection specimen $(P<0.02$, paired $t$-test $)$.

Table 1 Linear regression analysis of mitotic figures vs MIB-1 immunohistochemistry in biopsies and resection specimen

\begin{tabular}{|c|c|c|c|}
\hline \multicolumn{2}{|c|}{ Biopsies } & \multicolumn{2}{|c|}{ Resections } \\
\hline $\begin{array}{l}\text { Mitotic figures/10 high } \\
\text { power fields }\end{array}$ & $\begin{array}{l}\text { Mitotic figures } \\
\text { (corrected) }\end{array}$ & $\begin{array}{l}\text { Mitotic figures/10 high } \\
\text { power fields }\end{array}$ & $\begin{array}{l}\text { Mitotic figures } \\
\text { (corrected) }\end{array}$ \\
\hline \multicolumn{4}{|l|}{ MIB-1 (visually estimated) } \\
\hline $\begin{array}{l}\text { Slope } \mathrm{x}=0.423 \\
\rho=0.583 \\
P<0.00000582\end{array}$ & $\begin{array}{l}\text { Slope } \mathrm{x}=0.523 \\
\rho=0.472 \\
P<0.00040464\end{array}$ & $\begin{array}{l}\text { Slope } \mathrm{x}=1.204 \\
\rho=0.654 \\
P<0.0000001\end{array}$ & $\begin{array}{l}\text { Slope } \mathrm{x}=1.192 \\
\rho=0.514 \\
P<0.000098\end{array}$ \\
\hline \multicolumn{4}{|l|}{ MIB-1 (counted) } \\
\hline $\begin{array}{l}\text { Slope } \mathrm{x}=0.407 \\
\rho=0.546 \\
P<0.00002808\end{array}$ & $\begin{array}{l}\text { Slope } \mathrm{x}=0.514 \\
\rho=0.458 \\
P<0.00064135\end{array}$ & $\begin{array}{l}\text { Slope } \mathrm{x}=1.235 \\
\rho=0.644 \\
P<0.00000025\end{array}$ & $\begin{array}{l}\text { Slope } \mathrm{x}=1.260 \\
\rho=0.518 \\
P<0.0000830\end{array}$ \\
\hline \multicolumn{4}{|l|}{ MIB-1 (computed) } \\
\hline $\begin{array}{l}\text { Slope } \mathrm{x}=0.559 \\
\rho=0.461 \\
P<0.00058774\end{array}$ & $\begin{array}{l}\text { Slope } \mathrm{x}=0.707 \\
\rho=0.396 \\
P<0.0036890\end{array}$ & $\begin{array}{l}\text { Slope } \mathrm{x}=1.668 \\
\rho=0.592 \\
P<0.0000038\end{array}$ & $\begin{array}{l}\text { Slope } \mathrm{x}=1.69 \\
\rho=0.496 \\
P<0.0001847\end{array}$ \\
\hline
\end{tabular}

The linear regression plot for counted MIB-1 data and raw, uncorrected mitotic figure counts are shown in Figure 3.

\section{Discussion}

This paper confirms prior reports that the number of mitotic figures is higher in resection specimen of breast carcinomas than in core needle biopsies, and that this results in an increase in combined tumor grades ${ }^{3,4}$ in around $25 \%$ of carcinomas. ${ }^{5-11}$ However, our interpretation of these data is fundamentally different from the one advanced in some of these previous reports. On the basis of the observations in the present study, we have no reason to believe that the discrepancy in mitotic figure counts is due to the proposed sampling artifact, ${ }^{9}$ where biopsies are taken at random from anywhere within the tumor, and not necessarily from the proliferatively active tumor periphery. If this was the case, the percentage of MIB-1-positive tumor cells should also be much higher in resection specimen than in biopsies. However, neither our present study (Figure 2) nor results published by other authors ${ }^{18}$ suggest that this is the case. Also, our observations do not support the idea that the discrepancy in mitotic figure counts should be due to the limited size of the biopsies, which could 


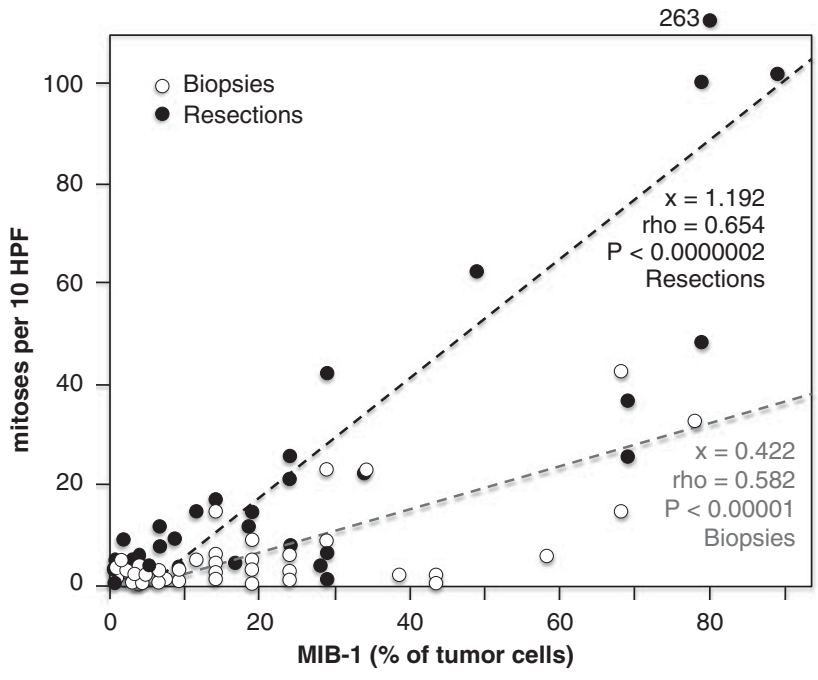

Figure 3 Linear regression analysis of mitotic figure counts (vertical axis) vs proliferative activity as assessed by MIB-1 immunohistochemistry (horizontal axis). Data are shown in full circles for resection specimen and in empty circles for biopsy material. Note the significant linear correlations for both types of tumor material, but which differ markedly by the slope of the regression lines. Data for slope (x) as well as $\rho$ - and $P$-values correspond to the data shown in Table 1. Shown in the plot are data for counted MIB-1 values and raw mitotic figure counts (not corrected for tumor/stroma rations in the respective high power fields). $P$-values were calculated using Spearman's linear regression analysis.

potentially preclude proper mitotic figure counting. ${ }^{9}$ In contrast, we observed no correlation between the size of the biopsies and the relative increase in mitotic figure counts from biopsies to resection specimen $(\rho=0.01859, \quad P=0.8952, \quad$ Spearman's analyses). Rather, we propose that the difference in mitotic figure counts between biopsies and resection specimen reflects a biological phenomenon, presumably secondary to intraoperative stress and specimen handling after removal from the breast. While biopsies are removed from the intact tumor and immediately immersed in formalin, resection specimen are exposed to intraoperative trauma and warm ischemia (due to ligation of feeding arteries to avoid bleeding), postoperative ischemia (during transport to radiology for specimen radiography and then on to pathology) and a poorly defined fixation delay in the formaldehyde container due to slow penetration of the fatty peritumoral tissue by the fixative. If one considers that formaldehyde penetrates tissues at a speed of $1 \mathrm{~mm} / \mathrm{h},{ }^{19}$ then the biopsy is fixed throughout within $15-30 \mathrm{~min}$, arresting all biological processes. In contrast, tumor cells in the resection specimen may benefit from many additional hours of more or less unimpaired biological activity before eventually being stopped by dwindling energy resources or by being fixed in the slowly advancing formaldehyde front. Once having trespassed the G0/G1 checkpoint, tumor cells are fatefully bound towards mitotic cell division. The cell cycle machinery will continue to work towards this aim even after removal of the tumor from the breast, and metabolic activities are maintained by intracellular glucose even under ischemic conditions, through the Warburg effect. However, in the absence of sustained energy supply, the gradually declining energy reserves will eventually arrest the cell cycle at the G2/M checkpoint; recent studies have shown that the mitotic machinery involves a metabolic sensor, the AMP-activated protein kinase ${ }^{20}$ which binds to various structures of the mitotic apparatus, including centrosomes, spindle poles and the spindle midzone. ${ }^{21}$ This mechanism is understood as an energy gauge that assures that the cell disposes of sufficient energy to complete faithful chromosome separation and thus exerts an important cytokinetic suppressor function. ${ }^{22}$ It is hence conceivable that mitotic activity continues in resection specimen up to the G2/M checkpoint, and that in the absence of sufficient energy reserves, tumor cells arrest in metaphase, where they are easily identified and counted by the diagnosing pathologist. The fact that we found significantly fewer post-metaphase figures (anaphase, telophase) in the resection specimen of our study ( $3 \%$ vs $7 \%$ in biopsies) would be consistent with this concept. Also, this idea would reconcile the apparent dilemma that despite markedly increased mitotic figure counts, MIB-1 immunoreaction seems not to go up in resection specimen (Figure 2a and ref. ${ }^{18}$ ). MIB-1 identifies all cycling cells, ${ }^{2}$ including cells in $\mathrm{S}$ and in G2 phase (which are not identifiable as proliferating cells in standard H\&E sections), and should hence not be altered by the progress of cells through the cell cycle towards mitosis.

In conclusion, we propose that the increased mitotic figure counts in resection specimen are secondary to poorly defined artifacts, which are probably quite variable from specimen to specimen and likely difficult to control in the clinical setting. Putative mechanisms include warm and cold ischemia and/or delayed formalin fixation. On the basis of the consideration raised above, we propose that in the clinical routine, chemotherapy treatment decisions should be based on the assessment of mitotic figures in the biopsies, and not-as proposed by most authors-in the resection specimen. ${ }^{9,10}$ This also suggest that in case of discrepancies, particular attention should be paid to the MIB-1-based proliferative activity, which appears not to be affected by the described phenomenon, but where other caveats, notably concerning interobserver variability of slide interpretation apply. ${ }^{23}$ In fact, similar recommendations have previously been advanced for other prognostic and predictive markers in breast cancer, such as hormone receptors ${ }^{24}$ and Her2/ $n e u,{ }^{25}$ and should raise the awareness that biopsy material of sufficient quantity and quality is the best possible basis for (neo-) adjuvant treatment decisions. Examination of the resection specimen should probably be limited to informations concerning tumor extension and spread (size, lymphovascular 
invasion, resection margins, presence of associated in situ lesions, etc).

\section{Acknowledgements}

The excellent technical assistance of the entire staff of the 'Grand Labo' at the Institute of Pathology, CHUV, Lausanne, is gratefully acknowledged.

\section{Disclosure/conflict of interest}

The authors declare no conflict of interest. This work is part of the doctoral dissertation and master thesis of Cornelia Schaper and Candice Rochat, respectively.

\section{References}

1 Amadori D, Volpi A, Maltoni R, et al. Cell proliferation as a predictor of response to chemotherapy in metastatic breast cancer: a prospective study. Breast Cancer Res Treat 1997;43:7-14.

2 Cattoretti G, Becker MH, Key G, et al. Monoclonal antibodies against recombinant parts of the Ki-67 antigen (MIB 1 and MIB 3) detect proliferating cells in microwave-processed formalin-fixed paraffin sections. J Pathol 1992;168:357-363.

3 Bloom HJG, Richardson WW. Histological grading and prognosis in breast cancer. Br J Cancer 1957;11:359-377.

4 Elston EW, Ellis IO. Pathological prognostic factors in breast cancer. I. The value of histological grade in breast cancer. Experience from a large study with longterm follow-up. Histopathology 1993;19:403-410.

5 Dahlstrom JE, Sutton S, Jain S. Histological precision of stereotactic core biopsy in diagnosis of malignant and premalignant breast lesions. Histopathology 1996;28:537-541.

6 Di Loreto C, Puglisi F, Rimondi G, et al. Large core biopsy for diagnostic and prognostic evaluation of invasive breast carcinomas. Eur J Cancer 1996;32A: 1693-1700.

7 Denley H, Pinder SE, Elston CW, et al. Preoperative assessment of prognostic factors in breast cancer. J Clin Pathol 2001;54:20-24.

8 Sharifi S, Peterson MK, Baum JK, et al. Assessment of pathologic prognostic factors in breast core needle biopsies. Mod Pathol 1999;12:941-945.

9 Harris GC, Denley HE, Pinder SE, et al. Correlation of histologic prognostic factors in core biopsies and therapeutic excisions of invasive breast carcinoma. Am J Surg Pathol 2003;27:11-15.

10 Badoual C, Maruani A, Ghorra C, et al. Pathological prognostic factors of invasive breast carcinoma in ultrasound-guided large core biopsies-correlation with subsequent surgical excisions. Breast 2005;14: 22-27.

11 Burge CN, Chang HR, Apple SK. Do the histologic features and results of breast cancer biomarker studies differ between core biopsy and surgical excision specimens? Breast 2006;15:167-172.

12 Lorgis V, Algros MP, Villanueva C, et al. Discordance in early breast cancer for tumour grade, estrogen receptor, progesteron receptors and human epidermal receptor-2 status between core needle biopsy and surgical excisional primary tumour. Breast 2011;20:284-287.

13 Amendoeira N, Apostolikas N, Bellocq JP, et al. Quality assurance guidelines for pathjology, in: European Guidelines for Quality Assurance in Breast Cancer Screening and Diagnosis (Perry N, Broeders M, deWolf C, Törnberg S, Holland R, vonKarsa L. (eds), 4th edn, Office of Official Publications of the European Communities: Luxembourg, 2006, pp288-289.

14 van Diest PJ, Baak JP, Matze-Cok P, et al. Reproducibility of mitosis counting in 2,469 breast cancer specimens: results from the Multicenter Morphometric. Mammary Carcinoma Project. Hum Pathol 1992;23: 603-607.

15 Tuominen VJ, Ruotoistenmäki S, Viitanen A, et al. ImmunoRatio: a publicly available web application for quantitative image analysis of estrogen receptor (ER), progesterone receptor (PR), and Ki-67. Breast Cancer Res 2010;12:R56.

16 Lehr HA, Hansen DA, Kussick S, et al. Assessment of proliferative activity in breast cancer: MIB-1 immunohistochemistry versus mitotic figure count. Hum Pathol 1999;30:1314-1320.

17 Weidner N, Moore DH 2nd, Vartanian R. Correlation of Ki-67 antigen expression with mitotic figure index and tumor grade in breast carcinomas using the novel "paraffin"-reactive MIB1 antibody. Hum Pathol 1994; 25:337-342.

18 Cavaliere A, Sidoni A, Scheibel M, et al. Biopathologic profile of breast cancer core biopsy: is it always a valid method? Cancer Lett 2005;218:117-121.

19 Start RD, Layton CM, Cross SS, et al. Reassessment of the rate of fixative diffusion. J Clin Pathol 1992; $451120-451121$.

20 Koh H, Chung J. AMPK links energy status to cell structure and mitosis. Biochem Biophys Res Commun 2007;362:789-792.

21 Vazquez-Martin A, Oliveras-Ferraros C, Menendez JA. The active form of the metabolic sensor: AMPactivated protein kinase (AMPK) directly binds the mitotic apparatus and travels from centrosomes to the spindle midzone during mitosis and cytokinesis. Cell Cycle 2009;8:2385-2398.

22 Vazquez-Martin A, López-Bonet E, Oliveras-Ferraros C, et al. Mitotic kinase dynamics of the active form of AMPK (phospho- AMPKalpha Thr172) in human cancer cells. Cell Cycle 2009;8:788-791.

23 Varga Z, Diebold J, Dommann-Scherrer C, et al. How reliable is Ki-67 immunohistochemistry in grade 2 breast carcinomas? A QA study of the Swiss working group of breast- and gynecopathologists. Plos One 2012;7:e37379.

24 Hammond ME, Hayes DF, Dowsett M, et al. American Society of Clinical Oncology/College of American Pathologists guideline recommendations for immunohistochemical testing of estrogen and progesterone receptors in breast cancer (unabridged version). Arch Pathol Lab Med 2010;134:e48-e72.

25 Wolff AC, Hammond ME, Schwartz JN, et al. American Society of Clinical Oncology/College of American Pathologists guideline recommendations for human epidermal growth factor receptor 2 testing in breast cancer. Arch Pathol Lab Med 2007;131:18-43. 